\title{
Identification of Weakly Nonlinear Systems Using Describing Function Inversion
}

\author{
H. Jalali • B.T. Bonab • H. Ahmadian
}

Received: 20 December 2009 / Accepted: 31 May 2010/Published online: 23 July 2010

(C) Society for Experimental Mechanics 2010

\begin{abstract}
In this paper describing functions inversion is used and the restoring force of a nonlinear element in a MDOF system is characterized. The describing functions can be obtained using linearized frequency response functions (FRFs). The response of the system to harmonic excitation forces at distinct frequencies close to the resonant frequency results in linearized FRFs. The nonlinear system can be approximated at each excitation frequency by an equivalent linear system. This approximation leads to calculation of the first-order describing functions. By having the experimental describing functions calculated and the system's responses corresponding to the nonlinear element (measured or interpolated), nonlinear parameter identification can be performed. Two numerical and experimental case studies are provided to show the applicability of this method.
\end{abstract}

Keywords MDOF system · Nonlinearity identification · Describing functions

\section{Introduction}

Several methods have been developed for identification of structural nonlinear systems since Ibanez [1] pioneered the idea at 1973. These methods can be categorized into

\footnotetext{
H. Jalali $(\bowtie)$

Iran University of Science and Technology-Arak Branch, Arak 38181-41167, Iran

e-mail: jalali@iust.ac.ir

B.T. Bonab $\cdot$ H. Ahmadian

Center of Excellence in Solid Mechanics and Dynamics, Iran University of Science and Technology,

Tehran 16868, Iran
}

linearization, time domain methods, frequency domain methods, time-frequency methods, modal methods, blackbox modeling and structural model updating. Kerschen et al. [2] presented a comprehensive literature survey on the above methods. From the time domain methods the restoring force surface method proposed by Masri and Caughey [3] has gained many attention for its simplicity and robustness.

For the weakly nonlinear systems usually the linearization method gives satisfactory results. Harmonic balance method [4] and describing functions method [5] can be used to obtain the equivalent linear system. Rice [6] used the equivalent linear system obtained by experimentally recorded structural responses and identified the parameters of a nonlinear system. Mayer and Link [7] linearized a nonlinear system by means of the harmonic balance method. They then identified the nonlinear stiffness and damping parameters by minimizing the differences between analytical and experimental frequency responses.

The aim of the describing functions method is to obtain an equivalent amplitude-dependent stiffness and damping coefficients for the nonlinear elements. Kul and Chen [8] proposed a method which can be used for obtaining the describing functions of hysteretic type nonlinearities. Their method is based on circular geometry shapes. The radius of the circular geometry shapes is a measure of the nonlinearities. Watanabe and Sato [9] used the first-order describing functions and linearized the nonlinear stiffness of a beam structure. Kuran and Ozguven [10] proposed an approach for quasi-linearization of MDOF systems by introducing describing functions for the cubic stiffness nonlinearity. Tanrikulu et al. [11] improved the method proposed by Kuran and Ozguven [10] by including a wider range of first-order describing functions. Besancon-Voda and Blaha [12] developed a multi-input describing function for the friction damping nonlinearity. 
Elizalde and Imregun [13] used the first order describing functions and obtained closed form expressions for frequency response functions of a nonlinear MDOF system. They considered cubic stiffness and friction damping nonlinearities. Ozer et al. [14] proposed a method for calculating describing functions by using the harmonic response of a MDOF nonlinear system. They then identified the restoring forces of the nonlinear element by curve fitting the plots of the obtaining describing functions $v s$. response amplitudes. The result will be a restoring force as a function of response amplitude. They also considered cubic stiffness and friction damping nonlinearities. For these types of nonlinearities the describing functions depend only upon the response amplitude level.

This paper considers identification of more general nonlinearities where their describing functions depend upon the response amplitude and frequency. Also the nonlinearity is identifies as a function of time. This is achieved by using the first-order describing functions obtained from experimental results. In fact, by measuring the systems response to a harmonic excitation force the linearized frequency response functions are obtained. Having the linearized frequency responses at each excitation frequency an equivalent linear system is constructed for the nonlinear system which leads to obtaining the describing functions. Performing an inversion of the describing functions finally results in the nonlinearity identification.

\section{Describing Functions: Definition and Inversion}

The describing functions method was first originated in control theory for solving nonlinear systems [15]. In this method each nonlinear element is replaced with a (quasi) linear element described by a function relating the elements' output to the input (i.e. a gain). The gain of the linear element is a function of its input amplitude. The form of the describing function therefore depends on the type of inputs. Sinusoidal input describing functions (SIDF) $[5,16]$ and random input describing functions (RIDF) [17] are two commonly used categories of describing functions. Following the SIDF method which is used in this paper is described in more details.

The SIDF approach can be used for characterization of nonlinear systems in frequency domain. As the name implies the system should be in periodic response condition. Consider the following SDOF nonlinear system,

$m \ddot{x}(t)+g(x(t), \dot{x}(t))=f \sin (\omega t)$

Assuming that the response of the system presented in equation (1) is sufficiently close to a pure sinusoidal i.e. $x(t) \approx a \sin (\tau)$, where $\tau=\omega t+\psi$ and there is little energy leaked to frequencies other than $\omega$, the nonlinear restoring force can be approximated by a quasi-linear amplitude dependent function as,

$g(x(t), \dot{x}(t)) \cong\left(n_{p}(a, \omega)+j n_{q}(a, \omega)\right) x(t)$

where $n_{p}$ and $n_{q}$ which are real and imaginary parts of an equivalent complex spring to be replaced with the nonlinear restoring force can be obtained by using the optimum quasi-linearization theory as [5],

$n_{p}(a, \omega)=\frac{1}{\pi a} \int_{0}^{2 \pi} g(a \sin (\tau), a \omega \cos (\tau)) \sin (\tau) d \tau$

$n_{q}(a, \omega)=\frac{1}{\pi a} \int_{0}^{2 \pi} g(a \sin (\tau), a \omega \cos (\tau)) \cos (\tau) d \tau$

The SIDF of many nonlinear restoring forces have been derived. A comprehensive list can be found in [5]. Equations (3) and (4) offer the describing functions of a known restoring force. The problem can be considered from the identification point of view as well. Given $n_{p}(a, \omega)$ and $n_{q}(a, \omega)$ one may be interested in finding the restoring force to which the given describing functions correspond. This procedure is called describing function inversion. In control theory polynomial based and piecewise-linear based describing function inversions are used. In structural dynamics Ozer et al. [14] identified the restoring forces of a nonlinear element in a MDOF system by curve fitting the plots of the obtained describing functions vs. response amplitudes. The result will be a restoring force as a function of response amplitude. They considered the cases where either $n_{p}(a, \omega)$ or $n_{q}(a, \omega)$ is zero. In this paper the describing functions of a MDOF system containing a nonlinear element is used and the restoring force of the nonlinear element is identified as a function of time.

\section{Problem Statement}

Equations governing the dynamic response of a nonlinear MDOF structure can be represented as,

$$
[M]\{\ddot{\mathrm{x}}\}+[K]\{x\}+\left\{F_{N L}(t)\right\}=\{F(t)\}
$$

In equation (5), $[M]$ and $[K]$ are $n \times n$ structural mass and stiffness matrices, respectively, $\{F(t)\}$ is the vector of external excitation and $\left\{F_{N L}(t)\right\}$ is a vector containing the nonlinearity effects. It is assumed that the system contains one nonlinear element between $i^{\text {th }}$ and $j^{\text {th }}$ DOFs. Therefore 
the nonlinear vector in equation (5) can be considered to have the following form,

$$
\begin{aligned}
& \left\{F_{N L}(t)\right\}=\left[0,0, \ldots, f_{N L}(z, \dot{z}), 0,0, \ldots,-f_{N L}(z, \dot{z}), \ldots, 0,0\right]^{T} \\
& z(t)=x_{i}(t)-x_{j}(t)
\end{aligned}
$$

Consider that the structure described in equation (5) is subjected to an external harmonic excitation force of the form $\{P\} \cos (\omega t)$. The excitation frequency belongs to a frequency interval containing the $s^{\text {th }}$ natural frequency of the structure, i.e. $\omega=\left[\omega_{s}-\sigma, \omega_{s}+\sigma\right]$; where $\sigma$ is chosen small and usually $\sigma<0.1 \omega_{s}$. Ahmadian and Zamani [17] showed that in such circumstances an equivalent linear system can be found for the nonlinear system at each excitation frequency. The governing equations of the equivalent linear system may be expressed as,

$$
[M]\{\ddot{x}\}+([K]+[\widetilde{K}])\{x\}=\{P\} \cos (\omega t)
$$

$[\widetilde{K}]$ is a complex matrix containing the linearized stiffness and damping effects of the nonlinear force $f_{N L}(z, \dot{z})$. The real part represents the linearized stiffness and the imaginary part models the linearised damping effects. $[\widetilde{K}]$ may be defined as,

$\widetilde{K}=\operatorname{zeros}(n, n), \widetilde{K}([i, j],[i, j])=\bar{k}\left[\begin{array}{cc}1 & -1 \\ -1 & 1\end{array}\right], \bar{k}=k_{r}+j k_{i}$

Provided that the linearized frequency responses of the nonlinear system are available, $k_{r}$ and $k_{i}$ can be obtained by solving the following equations in an iterative procedure [17],

$$
\begin{aligned}
& \left(\omega_{s}^{2}+\left[J_{\omega}\right]\{\Delta k\}-\omega^{2}\right)\{\bar{X}(\omega)\} \\
& -\left(\left[J_{\varphi}\right]\{\Delta k\}+\left\{\varphi_{s}\right\}\right)^{T}\{P\}\left(\left[J_{\varphi}\right]\{\Delta k\}+\left\{\varphi_{s}\right\}\right)=0 \\
& {\left[J_{\omega}\right]=\frac{\partial \omega_{s}^{2}}{\partial\{\Delta k\}}, \quad\left[J_{\varphi}\right]=\frac{\partial\left\{\varphi_{s}\right\}}{\partial\{\Delta k\}}, \quad\{\Delta k\}=\left[\Delta k_{r}, \Delta k_{i}\right]^{T},} \\
& \{\bar{X}(\omega)\}=\left\{X^{e x}(\omega)\right\}-\sum_{r=1, r \neq s}^{N} \frac{\left\{\varphi_{r}\right\}^{T}\{P\}\left\{\varphi_{r}\right\}}{\omega_{r}^{2}-\omega^{2}}
\end{aligned}
$$

where $\left\{X^{e x}(\omega)\right\}$ is the experimental frequency response vector. Linearized frequency response functions will be described in more details in next sections. In the following it will be shown that the equivalent linear springs, i.e. $k_{r}$ and $k_{i}$, are the describing functions introduced in previous section.

The structure is subjected to a harmonic excitation force with a frequency close to $s^{\text {th }}$ natural frequency; therefore at each excitation frequency the response can be approximated by using its $s^{\text {th }}$ normal mode as $\{X(t)\}=\left\{\widetilde{\phi}_{s}\right\} q(t)$. The normal mode $\left\{\widetilde{\phi}_{s}\right\}$ corresponds to the equivalent linear system obtained at that excitation frequency. Substituting the assumed response into equations (5) and (7), premultiplying the resultant equation into $\left\{\widetilde{\phi}_{s}\right\}^{T}$ and using the orthogonality properties of the mode shapes finally leads respectively to equations (11) and (12),

$$
\ddot{q}(t)+\omega_{s}^{2} q(t)+\left(\widetilde{\phi}_{s i}-\widetilde{\phi}_{s j}\right) f_{N L}(z, \dot{z})=\left(\left\{\widetilde{\phi}_{s}\right\}^{T}\{P\}\right) \cos (\omega t)
$$

$$
\begin{aligned}
\ddot{q}(t) & +\omega_{s}^{2} q(t)+\left(\widetilde{\phi}_{s i}-\widetilde{\phi}_{s j}\right)^{2}\left(k_{r}+j k_{i}\right) q(t) \\
= & \left(\left\{\widetilde{\phi}_{s}\right\}^{T}\{P\}\right) \cos (\omega t)
\end{aligned}
$$

In equation (11) $\mathrm{z}(\mathrm{t})=\left(\widetilde{\phi}_{\mathrm{si}}-\widetilde{\phi}_{\mathrm{sj}}\right) q(t) . \widetilde{\phi}_{s i}$ and $\widetilde{\phi}_{s j}$ are respectively the $i^{\text {th }}$ and $j^{\text {th }}$ members of $\left\{\widetilde{\phi}_{s}\right\}$. Comparing the last two equations, equations (11) and (12), shows that,

$f_{N L}(z, \dot{z}) \cong\left(k_{r}+j k_{i}\right) z(t)$

Comparing equations (2) and (13) indicates that the real and imaginary parts of the equivalent linear system, i.e. $k_{r}$ and $\mathrm{k}_{\mathrm{i}}$, can be considered as describing functions. $k_{r}$ and $k_{i}$ are obtained from the linearized frequency responses of the nonlinear system as was described in equations (9) and (10).

Equations (3), (4), (9) and (10) pave a foundation for identification of nonlinear systems using experimental results. First the linearized parameters $k_{r}$ and $k_{i}$ are identified using experimental frequency responses and by adopting equations (9) and (10). The linearized parameters are then substituted in equations (3) and (4). Solving these two equations simultaneously leads to identification of the nonlinear function $f_{N L}(z, \dot{z})$. Equations (3) and (4) needs $z(t)$ and its time derivative to be known. It is not always possible to measure this response coordinate; especially when the nonlinear element is a joint or a boundary condition. Elizalde and Imregun [13] measured this coordinate directly from the test experiment. In this paper this coordinate is obtained by using the equivalent linear system of equation (7).

The remaining sections of this paper go as follows. In next section the above described method are applied to a 2DOF system with a nonlinear element consisting of a cubic spring and a quadratic damping to show the capability of the method in simulated data. Next the method is evaluated using experimental results measured from test set-up of a clamped beam. Micro-slips develop at the clamped end at high excitation amplitudes. 


\section{Numerical Example}

A schematic of the two degrees-of-freedom system used in numerical example is shown in Fig. 1. The system which includes a nonlinear element $\mathrm{NL}$ is excited using a harmonic force. A model consisting of a softening spring and a quadratic damping is considered in this paper for nonlinear element NL. The capability of the method described in previous section in identification of this model is investigated here. The restoring force of nonlinear element NL is defined as,

$f_{N L}\left(x_{2}, \dot{x}_{2}\right)=k_{3} x_{2}(t)-k_{4} x_{2}(t)^{3}+c_{1} \dot{x}_{2}(t)+c_{2} \dot{x}_{2}(t)\left|\dot{x}_{2}(t)\right|$

The parameters of equation (14) are given in Table 1. The equations governing dynamic response of the system shown in Fig. 1 can be expressed as,

$$
\begin{gathered}
{\left[\begin{array}{cc}
m_{1} & 0 \\
0 & m_{2}
\end{array}\right]\left\{\begin{array}{l}
\ddot{x}_{1}(t) \\
\ddot{x}_{2}(t)
\end{array}\right\}+\left[\begin{array}{cc}
k_{1}+k_{2} & -k_{2} \\
-k_{2} & k_{2}
\end{array}\right]\left\{\begin{array}{l}
x_{1}(t) \\
x_{2}(t)
\end{array}\right\}} \\
+\left\{\begin{array}{c}
0 \\
f_{N L}\left(x_{2}, \dot{x}_{2}\right)
\end{array}\right\}=\left\{\begin{array}{l}
F \\
0
\end{array}\right\} \cos (\omega t)
\end{gathered}
$$

Equation (15) is solved numerically using a fourth order variable step Runge-Kutta method and the time domain response of two masses is obtained at different excitation frequencies. At each excitation frequency, simulated noise signals are added to the numerically calculated responses. This is done to investigate the robustness of the proposed method in identification of the nonlinear system parameters. The added noise signals are generated using randn function of MATLAB. Three cases of zero mean noise signals are considered. Their standard deviations are chosen to be $5 \%$, $10 \%$ and $15 \%$ of the maximum amplitude of the system response signals.

In order to calculate the frequency response functions (FRFs), first the time domain responses are low-pass filtered and only their first harmonic is kept, i.e. $x_{i}(t) \approx X_{i}\left(\omega t+\psi_{i}\right) i=1,2 . \psi_{\mathrm{i}}$ is the phase difference between excitation force and response signals. Having the

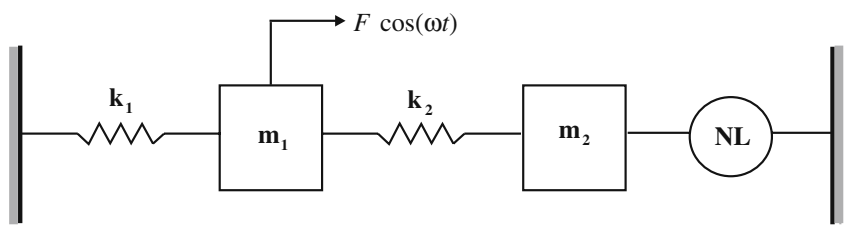

Fig. 1 Nonlinear $2 \mathrm{DOF}$ system, $\mathrm{m}_{1}=2 \mathrm{Kg}, \mathrm{m}_{2}=3 \mathrm{Kg}, \mathrm{k}_{1}=100 \mathrm{~N} / \mathrm{m}$, $\mathrm{k}_{2}=250 \mathrm{~N} / \mathrm{m}, \mathrm{F}=5 \mathrm{~N}$
Table 1 Exact and identified parameters of system with softening stiffness and quadratic damping

\begin{tabular}{lcccc}
\hline & $k_{3}(\mathrm{~N} / \mathrm{m})$ & $k_{4}\left(\mathrm{~N} / \mathrm{m}^{3}\right)$ & $c_{1}(\mathrm{~N} . \mathrm{s} / \mathrm{m})$ & $c_{2}\left(\mathrm{N.s} / \mathrm{m}^{2}\right)$ \\
\hline Exact & 120 & \multicolumn{1}{c}{800} & 3 & 1 \\
Identified (noise free) & 119.99 & 792.37 & 2.99 & 1.03 \\
Error (\%) & 0.0 & -0.95 & -0.30 & 3.04 \\
Identified (5\% noise) & 119.99 & 792.49 & 2.98 & 1.03 \\
Error (\%) & 0.0 & -0.93 & -0.47 & 3.63 \\
Identified (10\% noise) & 119.98 & 792.59 & 2.98 & 1.04 \\
Error (\%) & 0.0 & -0.92 & -0.64 & 4.22 \\
Identified (15\% noise) & 119.98 & 792.65 & 2.97 & 1.04 \\
Error (\%) & -0.01 & -0.91 & -0.80 & 4.81 \\
\hline
\end{tabular}

single harmonic response signals, the FRFs are obtained using the following equations,

$\alpha_{i 1}(\omega)=\frac{X_{i}}{F} e^{j \psi_{i}}, \quad i=1,2$,

It is worth mentioning that by band-pass filtering the response signals of nonlinear system, linearized FRFs are obtained. The linearized FRF of the nonlinear system is compared with the FRF obtained from the linear system in Fig. 2. The results shown in Fig. 2 correspond to the case of noise free responses.

By using the linearized frequency responses and employing equations (9) and (10), parameters of the equivalent linear system, i.e. $k_{r}$ and $k_{i}$, are identified at each excitation frequency. Having the parameters of the equivalent linear system available, the frequency response curve of nonlinear system can be regenerated for comparison purposes. This is done in Fig. 2 for the case of noise free responses. In this figure circles (o) show the regenerated frequency response curve. The good agreement between these two sets of the

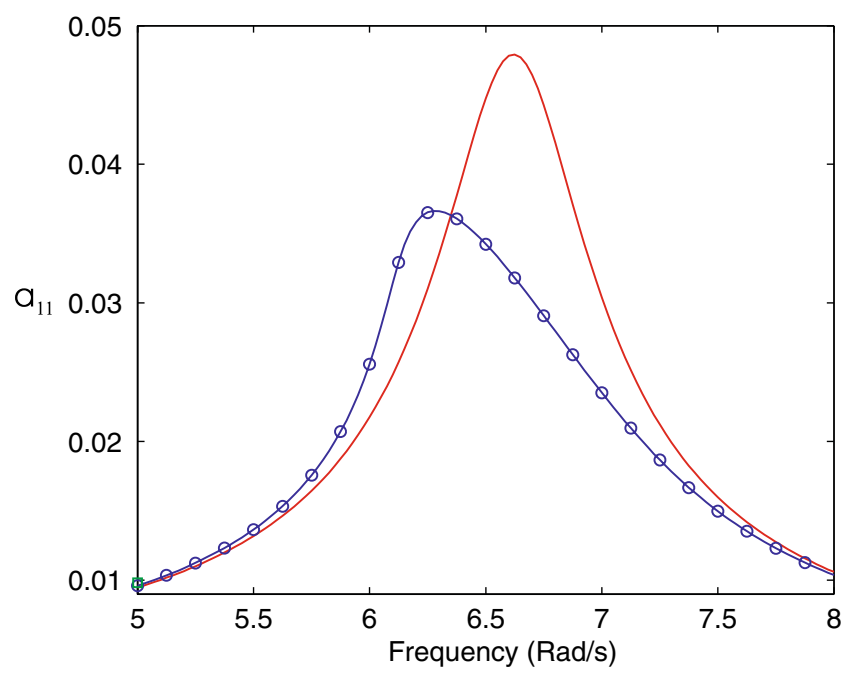

Fig. 2 (Color online) The FRF of linear system (red), the linearised FRF (blue) and the FRF obtained from equivalent linear system (o) 


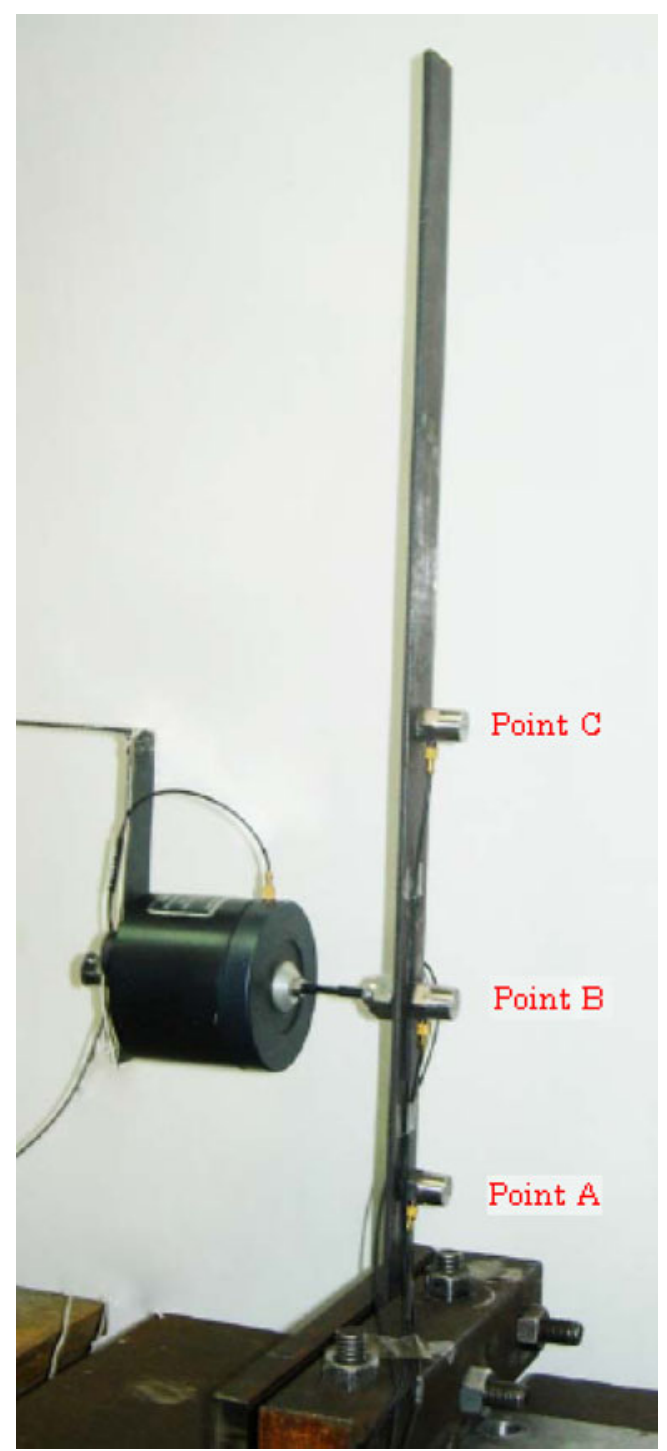

Fig. 3 Test set up

results show the accuracy in identification of the parameters of the equivalent linear system. Similar results are also obtained for the other cases of added simulated noise signals.

Up to this point the filtered response signals (i.e. the approximated single harmonic signal) and the parameters of the equivalent linear system $k_{r}$ and $k_{i}$, or describing functions, have been calculated at different excitation frequencies. Next we turn our attention to identify the parameters of the nonlinear restoring force $f_{N L}\left(x_{2}, \dot{x}_{2}\right)$. Substituting equation (14) into equations (3) and (4) results in the following equations,

$$
\begin{aligned}
& n_{p}\left(X_{2}, \omega\right)=\frac{1}{\pi X_{2}} \int_{0}^{2 \pi}\left\{k_{3} X_{2} \sin ^{2} \tau-k_{4}\left(X_{2}\right)^{3} \sin ^{4} \tau\right. \\
& \left.\quad+c_{1} \omega X_{2} \cos \tau \sin \tau+c_{2} \omega^{2}\left(X_{2}\right)^{2} \cos \tau \sin \tau|\cos \tau|\right\} d \tau
\end{aligned}
$$

$$
\begin{aligned}
& n_{q}\left(X_{2}, \omega\right)=\frac{1}{\pi X_{2}} \int_{0}^{2 \pi}\left\{k_{3} X_{2} \sin \tau \cos \tau-k_{4}\left(X_{2}\right)^{3} \sin ^{3} \tau\right. \\
& \left.\cos \tau+c_{1} \omega X_{2} \cos ^{2} \tau+c_{2} \omega^{2}\left(X_{2}\right)^{2} \cos ^{2} \tau|\cos \tau|\right\} d \tau
\end{aligned}
$$

It should be noted that $n_{p}\left(X_{2}, \omega\right)$ and $n_{q}\left(X_{2}, \omega\right)$ are respectively equal to $k_{r}$ and $k_{i}$ at corresponding frequencies. Performing the above integrals at each excitation frequency and after rearrangements, the results can be expressed in the form of a set of linear equations as $[\Gamma]\{y\}=\{b\}$, where:

$$
[\Gamma]=\left[\begin{array}{cccc}
1 & -\frac{3}{4}\left(X_{2}\right)_{\omega_{1}}^{2} & 0 & 0 \\
0 & 0 & \omega_{1} & \frac{8}{3 \pi}\left(X_{2}\right)_{\omega_{1}} \omega_{1}^{2} \\
\vdots & \vdots & \vdots & \vdots \\
1 & -\frac{3}{4}\left(X_{2}\right)_{\omega_{n}}^{2} & 0 & 0 \\
0 & 0 & \omega_{n} & \frac{8}{3 \pi}\left(X_{2}\right)_{\omega_{n}} \omega_{n}^{2}
\end{array}\right],
$$$$
\{y\}=\left\{\begin{array}{c}
k_{3} \\
k_{4} \\
c_{1} \\
c_{2}
\end{array}\right\},\{b\}=\left\{\begin{array}{c}
\left.k_{r}\right|_{\omega_{1}} \\
\left.k_{i}\right|_{\omega_{1}} \\
\vdots \\
\left.k_{r}\right|_{\omega_{n}} \\
\left.k_{i}\right|_{\omega_{n}}
\end{array}\right\}
$$

where $\left(X_{2}\right)_{\omega_{n}},\left.k_{r}\right|_{\omega_{n}}$ and $\left.k_{i}\right|_{\omega_{n}}$ are respectively amplitude of filtered response of mass $m_{2}$, real and imaginary parts of equivalent stiffness coefficient (or describing functions); all calculated at excitation frequency of $\omega_{n}$. Solving equation (19) finally results in unknown parameters of nonlinear restoring force, i.e. $\{y\}=[\Gamma]^{ \pm}\{b\} .[\Gamma]^{ \pm}$represents the pseudo-inverse. The exact and identified parameters are compared in Table 1 for three different cases of considered noisy responses.

Results presented in Table 1 shows that the method described in this paper is well able in identification of the nonlinear system parameters when the noise level is under $10 \%$. This indicates the robustness of the proposed method. In next section the method is verified using experimental results.

\section{Experimental Case Study}

A clamped-free beam as is depicted in Fig. 3 is used in this section. The beam contains nonlinear effects due to friction

Table 2 First four natural frequencies $(\mathrm{Hz})$

\begin{tabular}{lrrrr}
\hline & $\omega_{1}$ & $\omega_{2}$ & $\omega_{3}$ & \multicolumn{1}{c}{$\omega_{4}$} \\
\hline Experimental & 12.19 & 76.97 & 213.99 & 407.03 \\
Updated & 12.14 & 77.21 & 215.01 & 403.68 \\
Error (\%) & 0.35 & -0.31 & -0.48 & 0.82 \\
\hline
\end{tabular}




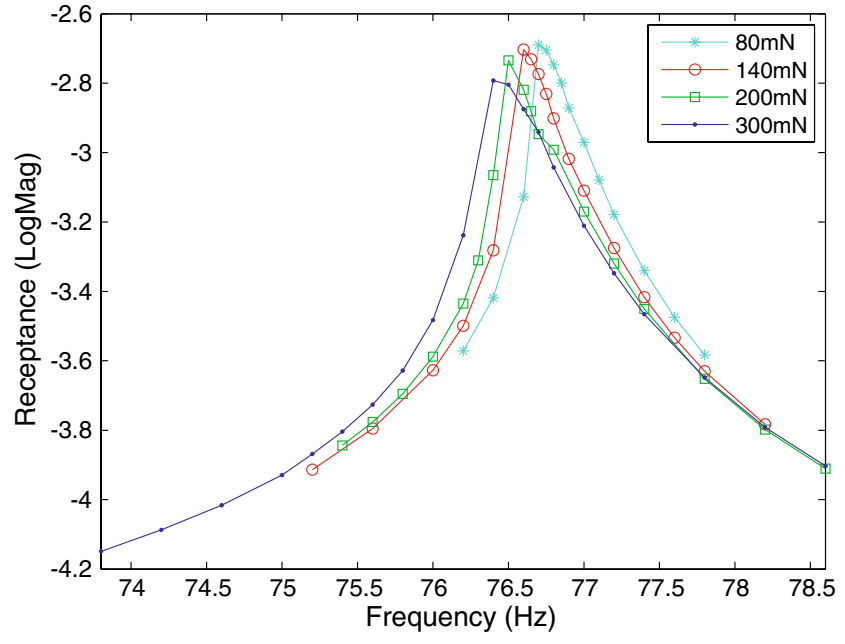

Fig. 4 Nonlinear frequency response curves at point B

contact at the clamped end. The material and geometric properties of the beam are: $\mathrm{E}=202.403 \mathrm{MPa}, \rho=7800 \mathrm{Kg} /$ $\mathrm{m}^{3}, \mathrm{~L}=0.527 \mathrm{~m}$ and $\mathrm{A}=116 \mathrm{~mm}^{2}$. The beam is excited by means of a $B \& K 4810$ mini-shaker at point $\mathrm{B}$. The applied force is measured using a $B \& K 8100$ force transducer. The beam response at points $\mathrm{A}, \mathrm{B}$ and $\mathrm{C}$ are measured by three $D J B A / 120 / V$ accelerometers. Points A, B and C are shown in Fig. 3.

Measurements are performed in two stages. In the first stage, the structure is excited using a low level random excitation force and the linear frequency response functions are measured. The natural frequencies of the structure extracted from the linear FRFs are tabulated in Table 2. In the second stage of experiments, a set of harmonic forces are used in order to extract the linearized frequency response functions of the nonlinear system. Four different excitation amplitudes are considered; namely 80, 120, 200 and $300 \mathrm{mN}$. It is believed that the nonlinear response of the structure is dominated by the micro-slips developing at the contact interface of clamped end [18]. Micro-slips are well excited near the second mode of the structure. Therefore keeping the excitation amplitude constant, the structure is excited at distinct frequencies near the second natural frequency. The steady state responses of points A, $\mathrm{B}$ and $\mathrm{C}$ and the applied force signals are measured. Having measured the excitation force and response signals, low-pass filtering the response signals and by employing equation (16), the linearized frequency response curves are obtained. The results corresponding to the accelerometer at point B are shown in Fig. 4.

The frequency responses shown in Fig. 4 indicate the softening effect due to the micro-slips developing at the clamped end. In the next section a Valanis model is considered for taking into account the effects of microslips and its parameters are identified using the method described in previous sections.

\section{Linear and Nonlinear Parameter Identification}

A FE model of the structure shown in Fig. 3 is built using 20 Euler-Bernoulli (2D) beam elements. In order to take into account the linear effects of the clamped end, the following stiffness matrix is added to the stiffness matrix of the beam at corresponding DOFs,

$$
[\bar{K}]=\left[\begin{array}{cc}
k_{w w} & k_{w t} \\
k_{w t} & k_{t t}
\end{array}\right]
$$
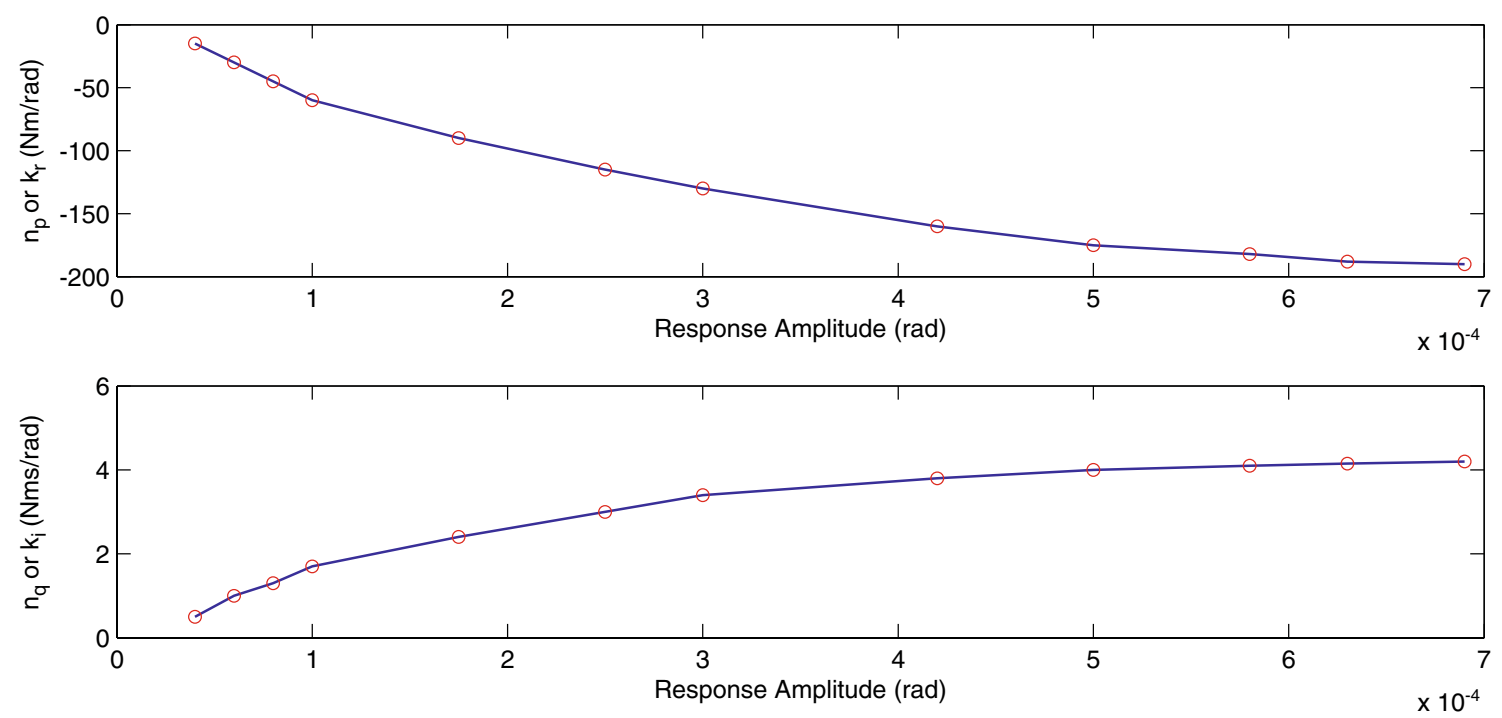

Fig. 5 Experimental describing functions 
Table 3 The parameters of Valanis model

\begin{tabular}{llll}
\hline $\mathrm{E}_{0}(N / m)$ & $\mathrm{E}_{\mathrm{t}}(\mathrm{N} / \mathrm{m})$ & $\lambda(1 / \mathrm{m})$ & $\kappa$ \\
\hline 123.70 & 58.46 & 21234.00 & 0.81 \\
\hline
\end{tabular}

where $k_{w w}, k_{t t}$ and $k_{w t}$ represent respectively the lateral stiffness, bending stiffness and coupling stiffness of the clamped end. These parameters are considered complex to take into account the damping effects of the contact interface. The linear stiffness parameters in equation (20) need to be identified. These parameters are identified by minimizing the differences between linear experimental FRFs and the FRFs obtained by using linear FE model. The experimental natural frequencies and those obtained from the updated FE model are compared in Table 2. These two sets of natural frequencies show a good agreement which is a result of accurate identified linear stiffness parameters.

Next, identification of the nonlinear characteristics of the clamped end is considered. A nonlinear FE model needs to be constructed for the beam shown in Fig. 3. The equation governing the nonlinear response of the beam is similar to equation (5). Mass matrix, stiffness matrix and the vector of external excitation forces are known from the linear FE model. A nonlinear force $f_{N L}$ is considered to represent the nonlinear effects of the micro-slips developing at the contact surface of the clamped end. The micro-slips correspond to the rotation DOF; therefore the vector of nonlinear forces $\left\{F_{N L}(t)\right\}$ to be substituted in equation (5) can be expressed as,

$\left\{F_{N L}(t)\right\}=\left[0, f_{N L}(\theta, \dot{\theta}), 0, \ldots, 0\right]^{T}$,

In equation (21), $\theta$ is the rotation DOF of the beam at the clamped end about the axis normal to the plane containing the beam and the shaker. In this paper a Valanis model is considered to govern the behavior of the micro-slips. The equation governing the restoring force of the Valanis model is shown in following,

$\dot{f}_{N L}(\theta, \dot{\theta})=\frac{E_{0} \dot{\theta}\left[1+\frac{\lambda}{E_{0}} \operatorname{sgn}(\dot{\theta})\left(E_{t} \theta-f_{N L}(\theta, \dot{\theta})\right)\right]}{1+\kappa \frac{\lambda}{E_{0}} \operatorname{sgn}(\dot{\theta})\left(E_{t} \theta-f_{N L}(\theta, \dot{\theta})\right)}$

where $E_{0}$ is the stiffness coefficient of the clamped end at sticking condition, $E_{t}$ denotes the slope of the slip motion, $\kappa$ controls the transition from stick to slip condition and $\lambda$ is characterized by the stick limit. The parameters of the Valanis model shown in equation (22) are identified in following using the method described in previous sections. It is worth mentioning that the experimental results corresponding to the excitation amplitude of $80 \mathrm{mN}$ are used in identification. Then, the identified model is validated by regenerating the experimental results corresponding to excitation amplitudes of $140 \mathrm{mN}$, $200 \mathrm{mN}$ and $300 \mathrm{mN}$.

First the experimental describing functions are calculated from the linearized frequency response function shown in Fig. 4. The describing functions will be substituted in equations (3) and (4) and the parameters of the Valanis model will be identified. In order to calculate the describing functions, a linear complex stiffness, as in equation (13), is replaced with the nonlinear Valanis model in equation (5). Then, using the frequency responses shown in Fig. 4 and employing equations (9) and (10) result in the describing functions (Fig. 5).

The next step in the identification of the Valanis model parameters is the calculation of the beam response states corresponding to the rotation DOF at the clamped end, i.e. $\theta(t)$ and $\dot{\theta}(t)$. It is not possible to measure these response states directly from the test set up, but they can be calculated by using the equivalent linear system and the measured response signals. Having obtained the equivalent linear system, or describing functions, the second mode shape $\left\{\widetilde{\phi}_{2}\right\}$ is calculated by using the FE model. Then, by
Fig. 6 The experimental (points) and predicted (lines) restoring force surfaces, $80 m N$ (o), $140 m N(\square), 200 m N$ $(\Delta)$ and $300 m N(\diamond)$

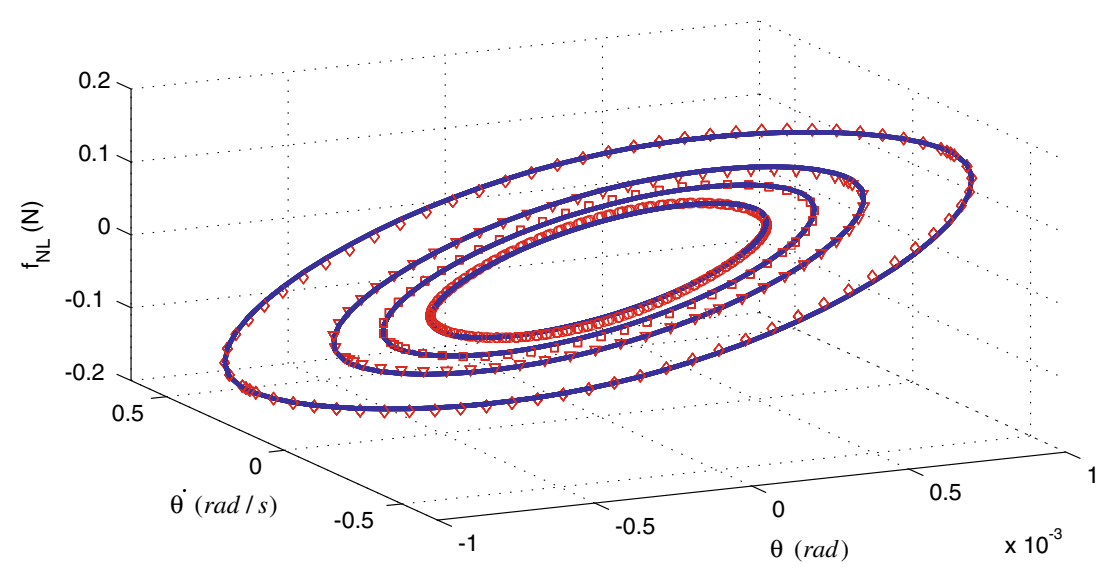


writing equation $\{X(t)\}=\left\{\widetilde{\phi}_{2}\right\} q(t)$ for the measured responses, $q(t)$ is calculated and using $q(t)$ the rotation DOF at the clamped end, i.e. $\theta(t)$, is obtained. Performing differentiation on $\theta(t), \dot{\theta}(t)$ is also known. It is worth mentioning that the structure is a weakly nonlinear system and is excited close to the second natural frequency; therefore it is assumed that the response is governed by the second mode, hence only this mode is used in calculation of $\theta(t)$. It also should be noted that because the equivalent linear systems, or describing functions, are amplitude dependent, different values for $\left\{\widetilde{\phi}_{2}\right\}$ are obtained at different excitation frequencies [18].

Up to this point the describing functions and the response states corresponding to the clamped end of the beam, i.e. $\theta(t)$ and $\dot{\theta}(t)$, are calculated. The final step in identification of the Valanis model parameters is as follows. First, a set of initial parameters are considered for Valanis model. At each excitation frequency the response states corresponding to rotation DOF at the clamped end, i.e. $\theta(t)$ and $\dot{\theta}(t)$, are known. Having known the Valanis model parameters and the response states, equation (22) is solved numerically and $f_{N L}$ is obtained. $f_{N L}$ is then substituted in the right hand side of equations (3) and (4) and the integrals are calculated numerically. The left hand side of equations (3) and (4) are describing functions, i.e. $k_{r}$ and $k_{i}$, which are known at each excitation frequency from the equivalent linear systems. The right hand side of these equations will result in describing functions too provided that the Valanis model parameters are correct. With the initial guess made for the Valanis model parameters, equations (3) and (4) usually results in residues. Applying the above procedure for all excitation frequencies, a vector of residues is obtained. The norm of this vector is minimized and the optimum Valanis model parameters are obtained. In this paper the genetic algorithm toolbox of Matlab is used and the vector of residues is minimized. Table 3 shows the optimum Valanis model parameters.

The parameters shown in Table 3 were obtained by using experimental results corresponding to the excitation amplitude of $80 \mathrm{mN}$. Using the Valanis model identified at $80 \mathrm{mN}$, the experimental results for other three excitation amplitudes, i.e 140, 200 and $300 \mathrm{mN}$, can be predicted for validation purposes. In Fig. 6 the experimental and predicted restoring forces obtained at different excitation amplitudes are compared. The contours in Fig. 6 correspond to the resonant frequency points of the frequency responses shown in Fig. 4. The experimental data in this figure are obtained by substituting measured responses into equation (11) and solving this equation for $f_{N L}$. The predicted restoring forces shown in Fig. 6 are obtained by substituting response states, i.e. $\theta(t)$ and $\dot{\theta}(t)$, into equation (21) and solving this equation numerically.
The results shown in Fig. 6 indicate that the Valanis model which was considered to represent the nonlinear effects of the micro-slips at the clamped end is a valid model. It can regenerate the experimental results used in identification and also is capable to predict the experimental results corresponding to higher excitation amplitudes with an acceptable accuracy.

\section{Conclusion}

Identification of weakly nonlinear MDOF systems was considered by using describing functions inversion. The system consists of a nonlinear element. An equivalent linear spring (to be replaced with nonlinear element) was identified at each excitation frequency and it was shown that real and imaginary parts of the equivalent linear stiffness are the describing functions of the nonlinear element restoring force. Using the describing functions inversion (numerically or analytically) the restoring force of the nonlinear element can be identified. This procedure was applied to two numerical and experimental case studies. In a numerical 2DOF system the nonlinear element was considered as combination of a cubic spring and a quadratic damping. The parameters of the nonlinear element were identified using the method described in this paper. An experimental clamped beam was also considered. The clamped end shows nonlinearity effects due to micro-slips developing at its contact interface. A Valanis model was considered to govern the micro-slip mechanism and its parameters were identified. The results showed that the proposed method is capable to identify the nonlinear systems with an acceptable accuracy.

\section{References}

1. Ibanez P (1997) Identification of dynamic parameters of linear and non-linear structural models from experimental data. Nucl Eng Des 25:30-41

2. Kerschen G, Worden K, Vakakis AF, Golinval JC (2006) Past, present and future of nonlinear system identification in structural dynamics. Mech Syst Signal Process 20:505-592. doi:10.1016/j. ymssp.2005.04.008

3. Masri SF, Caughey TK (1979) A nonparametric identification technique for nonlinear dynamic problems. J Appl Mech 46:433-447

4. Nayfeh AH, Mook DT (1979) Nonlinear Oscillations. WileyInterscience, New York

5. Gelb A, Vander Velde WE (1968) Multiple-input describing functions and nonlinear system design. McGraw-Hill Book Co., New York

6. Rice HJ (1995) Identification of weakly non-linear systems using equivalent linearization. J Sound Vib 185:473-481

7. Meyer S, Weiland M, Link M (2003) Modeling and updating of local non-linearities using frequency response residuals. Mech Syst Signal Process 17:219-226. doi:10.1006/mssp.2002.1563 
8. Kul YH, Chen CF (1962) A new method for evaluating the describing function of hysteresis-type nonlinearities. J Franklin Inst 273:226-241

9. Watanabe K, Sato H (1988) A modal analysis approach to nonlinear multidegrees-of-freedom systems. ASME J Vibrations Stress Reliab Des 189:110-410

10. Kuran B, Ozguven HN (1996) A modal superposition method for non-linear structures. J Sound Vib 189:315-339

11. Tanrikulu O, Kuran B, Ozguven HN, Imregun M (1993) Forced harmonic response analysis of non-linear structures. AIAA J 31:1313-1320

12. Besancon-Voda A, Blaha P (2002) Describing function approximation of a two-relay system configuration with application to Coulomb friction identification. Control Eng Pract 10:655-668. doi:10.1016/S0967-0661(02)00006-0

13. Elizaldea H, Imregun M (2006) An explicit frequency response function formulation for multi-degree-of-freedom non-linear sys- tems. Mech Syst Signal Process 20:1867-1882. doi:10.1016/j. ymssp.2005.12.009

14. Ozer MB, Ozguven HN, Royston TJ (2009) Identification of structural non-linearities using describing functions and the Sherman-Morrison method. Mech Syst Signal Process 23:30-44. doi:10.1016/j.ymssp.2007.11.014

15. Bogoliubov NN, Mitropolsky JA (1961) Asymptotic methods in the theory of non-linear Oscillations. Gordon and Breach, New York

16. Atherton DP (1982) Nonlinear control engineering. Van Nostrand Reinhold Co., New York

17. Gelb A, Warren RS (1973) Direct Statistical Analysis of Nonlinear Systems. AIAA J 11:689-694

18. Ahmadian H, Zamani A (2009) Identification of nonlinear boundary effects using nonlinear normal modes. Mech Syst Signal Process 23:2008-2018. doi:10.1016/j.ymssp.2008.07. 013 